\title{
Les droits sexuels et reproductifs à l'épreuve des religions dans la stratégie environnementale onusienne
}

Lucie Veyretout

\section{CpenEdition}

\section{Journals}

Édition électronique

URL : http://journals.openedition.org/rdr/952

DOI : $10.4000 /$ rdr.952

ISSN : 2534-7462

Éditeur

Presses universitaires de Strasbourg

\section{Édition imprimée}

Date de publication : 8 novembre 2016

Pagination : 157-167

ISBN : 978-2-86820-959-7

ISSN : 2493-8637

\section{Référence électronique}

Lucie Veyretout, «Les droits sexuels et reproductifs à l'épreuve des religions dans la stratégie environnementale onusienne ", Revue du droit des religions [En ligne], 2 | 2016, mis en ligne le 11 février 2020, consulté le 19 novembre 2020. URL : http://journals.openedition.org/rdr/952 ; DOI : https:// doi.org/10.4000/rdr.952 


\section{LES DROITS SEXUELS ET REPRODUCTIFS À l'ÉPREUVE DES RELIGIONS DANS LA STRATÉGIE ENVIRONNEMENTALE ONUSIENNE}

\section{Lucie VEYRETOUT}

Docteure en droit public de I'Université de Strasbourg

\section{RÉSUMÉ}

Dans les années 1970, la communauté internationale met en place une stratégie pour lutter contre les périls environnementaux. Dans ce cadre, des droits relatifs à la sexualité et à la reproduction y sont progressivement affirmés, notamment en lien avec l'une des préoccupations soulevées dès 1972 dans la Déclaration de Stockholm : la croissance démographique. Cependant, des objections issues de positions religieuses émanant d'États et d'autorités participant aux conférences environnementales sont soulevées face à la promotion de ces droits sexuels et reproductifs. Cet article en présente la teneur ainsi que leurs conséquences sur l'affirmation de ces droits au sein de la stratégie environnementale onusienne.

\section{ABSTRACT}

In the 1970's, the international community has set a strategy to fight against environmental hazards. In this context, the rights relating to sexuality and reproduction are gradually asserted, especially in connection with one of the concerns raised in 1972 and expressed in the Stockholm Declaration: the population growth. However, States and authorities participating in UN environmental conferences have raised religious driven objections to the promotion of sexual and reproductive rights. This paper presents such religious views and their consequences on the promotion of those rights within the UN environmental strategy. 
$\mathrm{D}$ ans les années 1970, face aux dégradations des écosystèmes et des ressources naturelles, les Nations unies mettent en place une stratégie et une politique pour lutter contre les périls environnementaux, lancées par la Déclaration de Stockholm de $1972^{1}$. À partir des années 1990, la promotion des droits des femmes y est développée en raison de liens remarqués entre l'environnement et les femmes. Selon la Déclaration de Rio sur l'environnement et le développement de 1992, « les femmes ont un rôle vital dans la gestion de l'environnement et le développement. Leur pleine participation est donc essentielle à la réalisation d'un développement durable ${ }^{2}$ » (principe 20). À la suite de cette déclaration, une attention particulière est systématiquement portée aux femmes au sein de la réflexion de la communauté internationale sur la protection de l'environnement. Les questions environnementales impliquent alors la promotion de l'égalité des sexes et de droits pour les femmes, notamment à travers leur participation, leur gestion des ressources naturelles, le contrôle de la fécondité. L'affirmation des droits relatifs à la sexualité et à la reproduction est liée à l'une des préoccupations soulevées dès 1972 dans la Déclaration de Stockholm : «l'augmentation naturelle de la population pose sans cesse de nouveaux problèmes pour la préservation de l'environnement ». Cependant, des objections issues de positions religieuses émanant d'États confessionnels et d'autorités religieuses participant aux conférences onusiennes sont soulevées face à la promotion de ces droits sexuels et reproductifs au sein des conférences environnementales. Quels sont ces obstacles et quelles en sont les conséquences sur l'affirmation des droits sexuels et reproductifs ? Depuis plusieurs années, la reconnaissance et le contenu des droits pour les femmes ne font pas l'objet d'une unanimité au sein des instances onusiennes. Selon les travaux de Denise Couture, on constate à partir des années 1990 une émergence de discours de nature religieuse mettant en avant « une vision religieuse des conditions des femmes » générant des « interactions » avec les droits des femmes, notamment de la part de participants musulmans et catholiques ${ }^{3}$. Cela est le cas

1. Déclaration finale de la Conférence des Nations unies sur l'environnement, Stockholm, 5-16 juin 1972. V. Kiss A., «Émergence de principes généraux du droit international et d'une politique internationale de l'environnement », in RENS I., Le droit international face à l'éthique et à la politique de l'environnement, Genève, Georg Éd., 1996, p. 19-33.

2. Le développement durable est défini comme « un processus de changement dans lequel l'exploitation des ressources, le choix des investissements, l'orientation du développement technique ainsi que le changement institutionnel sont déterminés en fonction des besoins tant actuels qu'à venir »: BrundtLand G. H., Notre avenir à tous, rapport de la Commission mondiale sur l'environnement et le développement, 1987, p. 14.

3. Couture D., « Droits des femmes et religions : analyse de quelques discours islamiques et catholiques », Studies in Religion / Sciences religieuses, 32/1-2, 2003, p. 6. 
pour l'affirmation et la promotion des droits sexuels et reproductifs dans le cadre de la stratégie environnementale. Les confrontations entre, d'un côté, la perspective religieuse soutenue par certains États et organisations non gouvernementales, et d'autre part, les droits sexuels et reproductifs promus par d'autres États et des mouvements défendant les droits des femmes se traduisent par une contestation de ces droits (1) conduisant à leurs remaniements (2).

\section{DES DROITS SEXUELS ET REPRODUCTIFS CONTESTÉS}

\subsection{DES DROITS RECONNUS COMME FONDAMENTAUX EN DROIT INTERNATIONAL}

Le programme d'action de la Conférence internationale sur la population et le développement du Caire de $1994^{4}$ énonce la définition et le contenu des droits sexuels et reproductifs dans son chapitre VII «droits et santé en matière de reproduction ». Selon ce texte, ces droits renvoient au «bien-être général, tant physique que mental et social, de la personne humaine, pour tout ce qui concerne l'appareil génital, ses fonctions et son fonctionnement», et à l'exercice d'une "vie sexuelle satisfaisante en toute sécurité »; ils impliquent l'accès à l'information et à des services de santé en matière de reproduction, de planification familiale (contraception, avortement), de sexualité, de maladies sexuellement transmissibles, ainsi que le choix en matière de procréation, et la fin des mutilations sexuelles féminines.

Ces droits sexuels et reproductifs sont considérés comme faisant partie des droits humains fondamentaux ${ }^{5}$. Ils sont affirmés dans l'acte final de la Conférence internationale des droits de l'homme de Téhéran dès $1968^{6}$ (liberté de choix des parents pour la taille de leur famille et «l'échelonnement des

4. Rapport de la Conférence internationale sur la population et le développement, Le Caire, 5-13 sept. 1994, New York, Nations unies, 1995, p. 38 (A/CONF.171/13/Rev.1).

5. Selon le programme d'action de 1994, «Les droits en matière de procréation correspondent à certains droits de l'homme déjà reconnus dans des législations nationales, des instruments internationaux relatifs aux droits de l'homme [...]. Ces droits reposent sur la reconnaissance du droit fondamental de tous les couples et des individus de décider librement et avec discernement du nombre de leurs enfants et de l'espacement de leurs naissances [...] et du droit de tous d'accéder à la meilleure santé en matière de sexualité et de reproduction. Ce droit repose aussi sur le droit de tous de prendre des décisions en matière de procréation $»$.

6. Proclamation de Téhéran, Acte final de la Conférence internationale des droits de l'homme, Téhéran, 22 avril-13 mai 1968, § 16 (A/CONF. 32/41 à 3). 
naissances ») et dans la Convention sur l'élimination de toutes les formes de discrimination à l'égard des femmes adoptée en $1979^{7}$, contraignante pour les États parties (article 12.1 relatif à l'accès des femmes aux services de planification familiale et article 16.1 e. sur les droits égaux en matière de choix procréatifs). Ces droits sont ensuite développés notamment dans les programmes d'action de la Conférence du Caire de 1994 et de la Conférence de Pékin de 1995 sur les femmes ${ }^{8}$.

Dans les années 1990, les textes relatifs aux droits des femmes - lesquels comprennent les droits sexuels et reproductifs - incluent la thématique environnementale et sont associés à la stratégie et à la politique environnementales internationales ${ }^{9}$. Ces dernières impliquent une réflexion sur la démographie au regard du rapport de proportionnalité entre population et ressources naturelles (Déclaration de Stockholm de 1972) et elles encouragent la mise en place de politiques démographiques : selon la Déclaration de Rio de 1992, " afin de parvenir à un développement durable et à une meilleure qualité de vie pour tous les peuples, les États devraient [...] promouvoir des politiques démographiques appropriées » (principe 8). Dans ce cadre, il y a une promotion des droits sexuels et reproductifs, valorisant le choix à égalité des hommes et des femmes en matière de procréation ainsi que l'accès aux informations et services permettant d'exercer ce droit, et encourageant les États à établir des programmes en vue d'instaurer des centres de soins génésiques et de planification familiale (points 24.2 g. et $24.3 \mathrm{du}$ programme Action 21).

Un rapport du Sénat français, établi en vue de la Conférence « Rio+20 » en 2012, traite des droits sexuels et reproductifs dans l'une de ses recommandations, au regard de la courbe de croissance de la population mondiale (estimation de 9 milliards d'humains vers 2050) et de son impact «sur l'environnement et sur notre bien-être " (selon un rapport de la Royal Society ${ }^{10}$ ) : ainsi, selon cette recommandation, "la Conférence "Rio+20" devra promouvoir le développement de l'accès à l'éducation pour tous et l'accès à la contraception »(recommandation 11$)^{11}$.

7. Texte disponible sur le site des Nations unies : http://www.un.org/womenwatch/daw/cedaw/ text/fconvention.htm [consulté le 3 juin 2016].

8. V. Gautier A., «Les droits reproductifs, une nouvelle génération de droits ? ", Autrepart, $\mathrm{n}^{\circ} 15,2000$, p. 167-180.

9. V. l'Agenda 21 adopté lors du sommet de la Terre de Rio de Janeiro en 1992, point 24.1.

10. Royal Society, People and the planet, London, April 2012.

11. Rossignol L., Rapport d'information fait au nom de la commission du développement durable, des infrastructures, de l'équipement et de l'aménagement du territoire, par le groupe de travail 
Cependant, la notion et le contenu des droits sexuels et reproductifs ne font pas l'unanimité au sein des instances onusiennes. Des participants en contestent la teneur au regard des doctrines religieuses.

\subsection{DES DROITS DIVERGEANT DES DOCTRINES RELIGIEUSES}

Les doctrines des grands monothéismes contiennent des règles relatives à la sexualité et à la reproduction. C'est en référence à ces règles que certains États, où la religion joue un rôle important dans la société ${ }^{12}$, marquent leurs désaccords avec certains droits sexuels et reproductifs promus au niveau international lors des conférences onusiennes ${ }^{13}$. En effet, alors que ces droits mettent en avant la liberté individuelle en matière d'union, de sexualité, de procréation ou d'orientation sexuelle, des règles religieuses posent un cadre reposant sur l'importance de la famille, du mariage hétérosexuel, de la maternité, de la sexualité dans le cadre du mariage ${ }^{14}$.

Selon la doctrine catholique, le mariage entre un homme et une femme est indissoluble, et la procréation et la fécondité y sont essentielles ( $\mathrm{n}^{\circ} 2249$, 2397 et 2398 du catéchisme de l'Église catholique de 1992) ; en conséquence, l'adultère, le divorce, la polygamie et l'union libre sont considérés comme « des offenses graves à la dignité du mariage » ( ${ }^{\circ} 2400$ du catéchisme de l'Église catholique). Les règles catholiques, prônant le «droit à la vie » de l'enfant dès sa conception, rejettent l'avortement direct jugé comme « une "pratique infâme" [...] gravement contraire à la loi morale » et, en tant que "délit contre la vie humaine », il est sanctionné "d'une peine canonique d'excommunication » ( $\mathrm{n}^{\circ} 2322$ du catéchisme de l'Église catholique). L'Église catholique désapprouve également l'homosexualité :

«S'appuyant sur la Sainte Écriture, qui les présente comme des dépravations graves (cf. Gn 19, 1-29; Rm 1, 24-27; 1 Co 6, 10 ; 1 Tm 1, 10), la Tradition a toujours déclaré que "les actes d'homosexualité sont intrinsèquement désordonnés" [...]. Ils sont contraires à la loi naturelle.

"Négociations internationales - Climat environnement ", sur Rio+20 : l'émergence d'un nouveau monde, Paris, Sénat, 2012, n 545, p. 47.

12. Tel est le cas notamment d'États dont la Constitution reconnaît une religion comme religion de l'État; nous pensons également au statut spécifique du Saint-Siège, observateur permanent aux Nations unies.

13. V. Couture D., art. cit.

14. Pour le catholicisme, V. les lettres encycliques Casti Connubii (1930) et Humanae vitae (1968) et l'exhortation apostolique Familiaris consortio (1981). 
Ils ferment l'acte sexuel au don de la vie.» (n² 2357 du catéchisme de l'Église catholique).

Pour sa part, la religion musulmane, qui admet dans une certaine mesure la contraception et l'avortement (en cas de danger de mort pour la mère, jusqu'à 120 jours ${ }^{15}$, met en avant la sexualité dans le cadre du mariage, la famille, la procréation et l'hétérosexualité ${ }^{16}$, le Coran énonçant notamment dans la sourate 23 Les Croyants : «1. Bienheureux sont certes les croyants [...] 5. [...] qui préservent leurs sexes [de tout rapport], 6. si ce n'est qu'avec leurs épouses ou les esclaves qu'ils possèdent, car là vraiment, on ne peut les blâmer ; 7. alors que ceux qui cherchent au-delà de ces limites sont des transgresseurs ${ }^{17}$.

En raison de ces règles religieuses, des États et autorités religieuses posent, lors des conférences internationales, des réserves et des déclarations interprétatives $^{18}$ à la reconnaissance et à la mise en ouvre de certains droits sexuels et reproductifs au niveau étatique. Cela fut notamment le cas lors de la Conférence internationale sur la population et le développement du Caire de 1994. Le Saint-Siège a émis une réserve sur l'ensemble du chapitre 7 «Droits et santé en matière de reproduction» du programme d'action, et d'autres pays ont également émis des déclarations et réserves sur ce chapitre tels que l'Argentine (concernant l'avortement), la République dominicaine (à propos du droit à la vie, de l'homosexualité, de la procréation hors mariage), l'Équateur (promotion de l'inviolabilité de la vie, protection de la famille), l'Égypte, le Guatemala, la République islamique d'Iran. Des réserves ont également été faites lors de la Conférence mondiale sur les femmes de Beijing en 1995 : « la majorité des [...] États du groupe islamo-catholique inscrivent des réserves au Programme d'action et elles concernent toutes, sans exception, la santé génésique et sexuelle des femmes ${ }^{19}$.

15. Chebel M., Vo «Avortement», in Dictionnaire encyclopédique du Coran, Paris, Fayard, 2009 , p. 57.

16. V. notamment Bellakhdar S., « La prescription de la sexualité en Islam », Topique, $\mathrm{n}^{\circ}$ 105, 2008, p. 105-116.

17. Le Coran, traduction en français du professeur Mouhammad Hamidullah : http://islamfrance.free.fr/doc/coran/sourate/23.html [consulté le 3 juin 2016].

18. Une réserve est la « déclaration unilatérale » d'un État qui « vise à exclure ou à modifier l'effet juridique de certaines dispositions du traité dans leur application à cet État »; une déclaration interprétative est la « déclaration unilatérale » d'un État qui « vise à préciser ou à clarifier le sens ou la portée que le déclarant attribue au traité ou à certaines de ses dispositions»: Pellet A., Troisième rapport sur les réserves aux traités. Annexe récapitulative - Guide de la pratique, New York, Nations unies, 1998, p. 1 et 3.

19. Couture D., art. cit., p. 11-12. 
À titre d'illustration, nous pouvons citer la déclaration lors de cette conférence du représentant du Saint-Siège et celle de la délégation du Brunei Darussalam (dont l'islam est la religion officielle) :

«Le Saint-Siège ne peut interpréter des termes tels que "le droit des femmes à être maîtresses de leur sexualité", "le droit des femmes à être maîtresses... de leur fécondité" ou "les couples et les individus", autrement que comme se référant à la pratique réfléchie de la sexualité dans le mariage. [...] Le Saint-Siège n'approuve aucune forme de législation qui confère à l'avortement une reconnaissance légale. [...] Le Saint-Siège n'approuve absolument pas le recours à la contraception ni l'emploi de préservatifs comme mesures de planification de la famille. $»^{20}$

\section{Pour l'État du Brunei Darussalam :}

«Les droits génésiques doivent s'appliquer uniquement aux couples mariés formés par l'union entre un homme et une femme " et « l'adoption du paragraphe 96 ne signifie pas que le Gouvernement du Brunei Darussalam approuve la promiscuité sexuelle [...] ou un comportement sexuel qui soit synonyme d'homosexualité et de lesbianisme. $»^{21}$

Les droits sexuels et reproductifs font également l'objet de discussions et de contestations dans le cadre des conférences environnementales. Cela a conduit dernièrement, lors de l'élaboration de la stratégie environnementale durant la Conférence sur le développement durable «Rio+20» de 2012, à des remaniements de ces droits.

\section{DES DROITS SEXUELS ET REPRODUCTIFS REMANIÉS}

\subsection{DES POSITIONS OPPOSÉES LORS DE L'ÉLABORATION DE LA STRATÉGIE ENVIRONNEMENTALE}

Dans le cadre des discussions sur la stratégie environnementale entre les États parties au sein des conférences onusiennes, des positions différentes se dessinent à propos des droits sexuels et reproductifs, quant à leur nature, leur composition et leur utilité dans le cadre de la protection de l'environnement. Une position consiste à s'intéresser à la promotion des droits des femmes dans

20. V. le Rapport de la quatrième conférence mondiale sur les femmes: Beijing, 4-15 septembre 1995, New York, Nations unies, 1996, p. 170-171 (A/CONF.177/20/Rev.1).

21. Ibid., p. 164. 
le cadre environnemental ainsi qu'à l'enjeu démographique (accroissement de la population) dans le développement durable ; cette position met en avant les droits sexuels et reproductifs. Une autre position, regroupant notamment des pays où la religion tient une place importante, réagit en défendant « le droit à la vie » et la famille, et en remettant en cause la nécessité d'une politique démographique comme réponse au développement durable. L'encyclique du Pape Laudato si de 2015, relative aux défis écologiques, énonce ce point de vue de la compatibilité entre l'accroissement de la population et les ressources naturelles. Selon le point 50 de l'encyclique:

«Au lieu de résoudre les problèmes des pauvres [...] certains se contentent seulement de proposer une réduction de la natalité. Les pressions internationales sur les pays en développement ne manquent pas, conditionnant des aides économiques à certaines politiques de "santé reproductive". Mais s'il est vrai que la répartition inégale de la population et des ressources disponibles crée des obstacles au développement et à l'utilisation durable de l'environnement, il faut reconnaître que la croissance démographique est pleinement compatible avec un développement intégral et solidaire. »

Les débats de la dernière conférence onusienne sur le développement durable « Rio+20 » de 2012 font ressortir ces différentes positions. Des États s'y sont prononcés en faveur d'un approfondissement des droits des femmes et des droits sexuels et reproductifs au niveau environnemental : par exemple, la ministre islandaise de l'Environnement a "plaidé en faveur de l'égalité entre les hommes et les femmes en tant que condition essentielle du développement durable », et le ministre de la Planification économique et sociale des Philippines a estimé que "l'égalité entre les sexes, l'autonomisation des femmes, les droits reproductifs et les droits de succession sont également des domaines où les efforts doivent être renforcés ${ }^{22}$.

D'autres États se sont en revanche prononcés défavorablement sur les droits sexuels et reproductifs dans le cadre de la stratégie environnementale :

« Le Vatican, en tant qu'observateur, la Russie, le Honduras, la République dominicaine, le Nicaragua, le Chili, la Syrie, l'Égypte, et le Costa Rica " ont estimé, à propos de "la mention "droits reproductifs" du brouillon final de l'accord issu de la conférence de l'ONU sur le développement durable Rio+20 [...] qu'ils ne comprenaient pas le rapport

22. «Rio+20 : les chefs d'État et de gouvernement de la planète renouvellent leur engagement politique en faveur du développement durable », 22 juin 2012 : http://www.un.org/News/ fr-press/docs/2012/ENVDEV1310.doc.htm [consulté le 3 juin 2016]. 
entre droits reproductifs des femmes et développement durable, que cette notion va à l'encontre de législations et constitutions nationales et qu'ils devaient protéger les droits des enfants à naître et le droit à la vie. $»^{23}$

Cela a abouti à certains remaniements des droits sexuels et reproductifs qui voient leur promotion varier au sein des instances onusiennes.

\subsection{UNE PROMOTION VARIABLE DES DROITS SEXUELS ET REPRODUCTIFS}

Dans le cadre de la conférence environnementale de 2012 réunissant les États membres de l'ONU, les droits sexuels et reproductifs ont fait l'objet de certains remaniements suite aux prises de position notamment de certains États ${ }^{24}$. Ces remaniements consistent tout d'abord en un changement de vocabulaire, avec l'emploi de l'expression «santé sexuelle et de la procréation » au lieu de « droit » à la santé sexuelle et de la procréation, et $\mathrm{du}$ verbe «promouvoir » au lieu d' « assurer ${ }^{25}$. Ils portent également sur la place octroyée à ces droits, avec par exemple la non-reconnaissance des « droits reproductifs des femmes comme un aspect central de l'égalité des sexes et du développement durable dans le document final de Rio+20 ${ }^{26}$.

Ces remaniements ont suscité la déception des organisations défendant les droits des femmes, lesquelles ont le sentiment « de s'épuiser à sauvegarder leurs acquis précédents ${ }^{27}$ et qu'il n'y a pas de progrès autour des droits des femmes ${ }^{28}$, ainsi que des représentants de certains États : le Premier

23. Moreno R., « Rio+20 : la mention "droits reproductifs" retirée de l'accord final », Égalité, dossier «Femmes et environnement : les enjeux des inégalités de genre », 2012, p. 28.

24. V. Moreno R., art. cit. et Nicolas Y., « RIO+20 : les femmes défendent leur pleine participation au développement durable ", Égalité, dossier « Femmes et environnement : les enjeux des inégalités de genre», 2012, p. 30.

25. Nicolas Y., «Rio+20 : les femmes défendent leur pleine participation à la réalisation d'un développement durable », Adéquations, 21 juin 2012 : http://www.adequations.org/spip. php?article1842 [consulté le 3 juin 2016] ; «Analysis Paper on the Rio+20 Process and Outcome Document » : http://www.dawnnet.org/feminist-resources/content/rio20-analysispaper-rio20-process-and-outcome-document [consulté le 3 juin 2016].

26. Groupe MAJEur FEMmes, «Rio+20 : Nous ne sommes pas passés au futur dont nous avons besoin », Égalité, dossier « Femmes et environnement : les enjeux des inégalités de genre $», 2012$, p. 24-25.

27. «Rio+20, Conférence mondiale des Nations unies sur le développement durable, 20-22 juin 2012 », Adéquations, 29 juin 2012 : http://www.adequations.org/spip.php?article1509 [consulté le 3 juin 2016].

28. «Les résultats de Rio+20 : les accords qui en émanent et leurs implications pour l'évolution des droits des femmes », AWID, 19 oct. 2012 : http://www.awid.org/fr/nouvelles-et-analyse/ 
ministre du Danemark, «a regretté que l'importance des droits reproductifs ne soit pas assez reconnue dans le projet de document final de Rio+20 » et le représentant du Nicaragua a estimé que « les pays ne devraient pas revenir sur des thèmes centraux qui avaient été débattus lors du Sommet Planète Terre, en 1992 à Rio » comme «les droits reproductifs » ${ }^{29}$.

La promotion des droits sexuels et reproductifs reste toutefois au cœur des programmes liés aux problématiques environnementales des instances onusiennes $^{30}$. Dans son rapport Une vie de dignité pour tous ${ }^{31}$ de 2013, le secrétaire général de l'ONU s'intéresse à la garantie des droits sexuels et reproductifs (point 27). Il énonce que «les femmes et les filles doivent accéder sur un plan d'égalité [...] à la gamme complète des services de santé, y compris dans les domaines de la santé sexuelle et de la procréation et des droits en matière de procréation » (point 85) et qu'il faut prendre des mesures pour «assurer la santé de la procréation et l'exercice des droits des femmes en matière de procréation » (point 87). En 2014, le groupe de travail mis en place conformément au paragraphe 248 du document final de la Conférence des Nations unies sur le développement durable «L'avenir que nous voulons» de 2012, a proposé dans son rapport des « objectifs de développement durable de portée mondiale devant être adoptés par l'Assemblée générale», parmi lesquels figurent les droits sexuels et reproductifs ${ }^{32}$. Ces droits sont énoncés dans les dix-sept objectifs de développement durable (ODD) adoptés en septembre 2015 par les États membres des Nations unies, dans l'objectif 3 « Accès à la santé. Donner aux individus les moyens de mener une vie saine et promouvoir le bien-être de tous à tous les âges " : "D'ici à 2030, assurer l'accès de tous à des services de soins de santé sexuelle et procréative, y compris à des fins de planification familiale, d'information et d'éducation, et la prise en compte de la santé procréative dans les stratégies et programmes nationaux»; et dans l'objectif 5 "Égalité entre les sexes.

les-resultats-de-rio20-les-accords-qui-en-emanent-et-leurs-implications-pour [consulté le 3 juin 2016].

29. «Rio+20 : les chefs d'État et de gouvernement de la planète renouvellent leur engagement politique en faveur du développement durable», 22 juin 2012 : http://www.un.org/News/ fr-press/docs/2012/ENVDEV1310.doc.htm [consulté le 3 juin 2016].

30. V. Nicolas Y., «L'égalité femmes-hommes dans le processus post 2015 », Adéquations, 3 juin 2013 : http://www.adequations.org/spip.php?article2017 [consulté le 3 juin 2016].

31. Une vie de dignité pour tous : accélérer les progrès dans la réalisation des objectifs du Millénaire pour le développement et dans la définition du programme de développement des Nations Unies pour l'après-2015, Rapport du secrétaire général, Assemblée générale des Nations unies, 26 juill. 2013 (A/68/202).

32. Rapport du Groupe de travail ouvert de l'Assemblée générale sur les objectifs de développement durable, Assemblée générale des Nations unies, 12 août 2014 (A/68/970). 
Parvenir à l'égalité des sexes en autonomisant les femmes et les filles» : «Assurer l'accès de tous aux soins de santé sexuelle et procréative et faire en sorte que chacun puisse exercer ses droits en matière de procréation, ainsi qu'il a été décidé dans le Programme d'action de la Conférence internationale sur la population et le développement et le Programme d'action de Beijing et les documents finals des conférences d'examen qui ont suivi $»^{33}$.

Alors que face aux défis environnementaux, les grandes religions monothéistes s'engagent largement en faveur de la protection de l'environnement, des éléments de leurs doctrines se heurtent à la promotion de certains droits sexuels et reproductifs dans le cadre de la stratégie environnementale au sein des instances onusiennes. Ce sont alors des systèmes de valeurs différents qui se font face, portant sur la sexualité et l'humanité, mais également sur les réponses possibles pour un développement durable, et qui confrontent des systèmes de droits - celui des droits fondamentaux du droit international et celui des normes religieuses. Ces différences ne doivent pas occulter cependant des possibilités de convergence à travers des évolutions des positions des autorités religieuses et des États et l'approfondissement d'une réflexion internationale sur le rapport entre croissance démographique et développement durable.

33. Objectifs de développement durable : 17 objectifs pour transformer notre monde : http:// www.un.org/sustainabledevelopment/fr [consulté le 3 juin 2016]. 